Copyright 2001 American Association of Physics Teachers (AIP Publishing). This article may be downloaded for personal use only. Any other use requires prior permission of the author and AIP Publishing.

The following article appeared as: Penner, A.R. (2001). The physics of golf: The optimum loft of a driver. American Journal of Physics, 69(5), 563-568, and may be found at http://dx.doi.org/10.1119/1.1344164 


\title{
The physics of golf: The optimum loft of a driver
}

\author{
A. Raymond Penner \\ Physics Department, Malaspina University-College, Nanaimo, British Columbia, Canada
}

(Received 27 March 2000; accepted 9 November 2000)

\begin{abstract}
The impact between a clubhead and a golf ball along with the resulting flight and run of the ball after landing is considered. The clubhead loft which results in the maximum drive distance and its dependence on the initial clubhead speed is then determined. (C) 2001 American Association of Physics Teachers.
\end{abstract}

[DOI: $10.1119 / 1.1344164]$

\section{INTRODUCTION}

Connecting physics to sports is an effective way to catch the interest of students. As an example, consider the problem of determining how to launch sports projectiles so as to maximize the distance they travel. In the case of a constant launch speed, the elementary treatment that disregards air drag shows that a launch angle of $45^{\circ}$ will lead to the maximum range. However, the elementary treatment breaks down if the projectile speed becomes too great, as it does in many sports. The magnitude of the air drag rises quickly with the speed of the projectile, which results not only in a reduced range but also in lowering the optimum launch angle required for maximum range. In addition, if the ball has significant spin, the air flow around the ball will exert an additional force perpendicular to the direction of travel. In the case of backspin, this effect results in a lifting force which both increases the range of the projectile and further lowers the optimum launch angle. The effect of air drag and lift on a spinning baseball has previously been considered in the literature. ${ }^{1-4}$ As an example, in the case of a typical homerun hit baseball with a launch speed of $50 \mathrm{~m} / \mathrm{s}$ and backspin of $1800 \mathrm{rpm}$, the critical launch angle is found to be lowered to approximately $35^{\circ}$. $^{1}$

Previous papers have explored the flight of a launched golf ball. Erlichson ${ }^{5}$ considered golf ball trajectories for linear, with and without lift, and quadratic air drag and determined the dependence of the range of the ball on the launch angle for the various drag models. Bearman and Harvey ${ }^{6}$ measured the lift and drag coefficients for both conventionally dimpled and hexagonally dimpled golf balls in wind tunnel tests and used these values to determine how the range depended on launch speed, launch angle, and the initial spin of the ball. MacDonald and Hanzely ${ }^{7}$ used the Bearman and Harvey coefficients to determine that the optimum launch angle of a golf ball driven at a constant launch speed of $61.0 \mathrm{~m} / \mathrm{s}$ and backspin of $3500 \mathrm{rpm}$, typical values for a ball hit with a driver, is $23^{\circ}$.

This paper will specifically consider the problem of launching a golf ball with a golf club and will determine the clubhead loft which leads to the maximum drive distance. This differs from the analysis of MacDonald and Hanzely in that when using a golf club to launch a golf ball, changing the loft of the club will not only change the launch angle, but it will also change both the launch speed and the backspin of the golf ball. Also, it is important to distinguish between the distance the ball travels through the air, or what in golf parlance is referred to as the carry, and the overall drive, which includes the run of the ball after it lands. When using a driver, it is the total overall drive distance that a golfer wishes to maximize. The theoretical analysis will therefore first consider the impact of the golf ball with the clubhead to determine the dependence of launch speed, launch angle, and backspin on clubhead loft. Then, by using the calculated launch parameters, the flight of the golf ball and its carry, along with the subsequent run, will be determined. The loft of the clubhead which leads to maximum overall drive distance will then be found.

\section{THEORY}

\section{A. Impact with the clubhead}

The collision between a golf club and a golf ball is a very violent event. The actual contact time is less than $0.5 \mathrm{~ms}$, during which the ball accelerates from rest up to speeds which can exceed $200 \mathrm{~km} / \mathrm{h}$. The forces involved are large, with values exceeding $5 \mathrm{kN}$, and the apparently solid ball is deformed significantly as can be seen in the flash photographs shown by Cochran and Stobbs. ${ }^{8}$ However, even this extreme event is analyzed, as with other collisions, by the laws of classical mechanics.

One surprising result which greatly simplifies the problem is that the effect of the club shaft during the collision can be ignored as the force that the shaft exerts during the collision is negligible as compared to the impact force. This was demonstrated by Cochran and Stobbs where a clubhead was hinged on a shaft and swung in the typical manner. The performance of the club and flash photographs showed that the collision with the golf ball was not significantly affected by this unique shaft. The clubhead will therefore be treated as a free body during the impact with the ball.

In order to analyze the effect of the loft of the clubhead on the carry and drive of a golf ball, simple models of the clubhead and the golf ball with be used. The clubhead will be modeled as a thin plate of mass $M$ and loft $\theta$ moving at a speed of $v_{c i}$ in the horizontal direction at the moment of impact. The golf ball will be modeled as a uniform solid sphere of mass $m$, radius $r$, and moment of inertia $I$ $=\left(\frac{2}{5}\right) m r^{2}$. The point of impact of the collision will be taken to occur at the center of mass of the plate as shown in Fig. 1(a) and any twisting of the clubhead during the collision will be neglected. The analysis of the collision will be done with respect to axes parallel and normal to the face of the clubhead. The components of the final velocities along these axes along with the final angular velocity of the golf ball are shown in Fig. 1(b).

The impact of a golf club with a golf ball has been previously considered by Daish ${ }^{9}$ and Jorgensen. ${ }^{10}$ Their analysis considered the forces and torques acting on the golf ball 


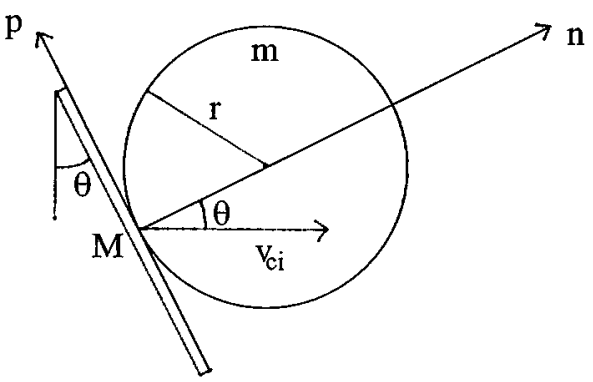

(a)

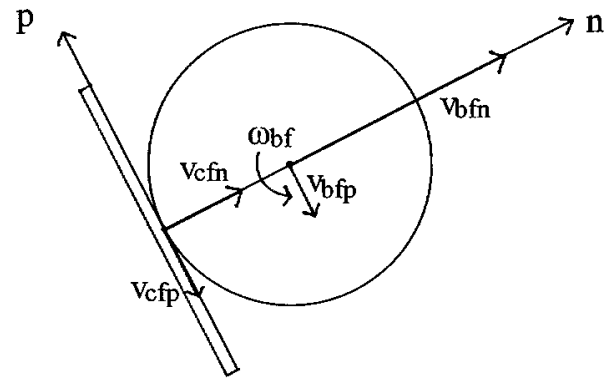

(b)

Fig. 1. (a) A clubhead of mass $M$, loft $\theta$, and speed $v_{c i}$ before impact with a golf ball of mass $m$ and radius $r$. (b) The velocity components of the clubhead and golf ball after impact.

during impact. The resulting equations, along with the conservation of linear momentum, allowed the launch speed, launch angle, and initial spin of the golf ball to be determined for a given initial clubhead speed and loft. However, the collision can also be analyzed without reference to the forces and torques, by simply considering, in addition to the conservation of linear momentum, the conservation of angular momentum. Conservation of linear momentum along the normal and parallel axes and conservation of angular momentum about the impact point lead to the following equations:

$$
\begin{aligned}
& M v_{c f n}+m v_{b f n}=M v_{c i} \cos \theta, \\
& M v_{c f p}+m v_{b f p}=-M v_{c i} \sin \theta,
\end{aligned}
$$

and

$$
I \omega_{b f}+m v_{b f p} r=0,
$$

where $v_{c f p}$ and $v_{c f n}$ are the velocity components of the clubhead after impact, and $v_{b f p}, v_{b f n}$, and $\omega_{b f}$ are the velocity components of the golf ball after impact.

Energy is lost during the collision as the ball becomes seriously deformed and the forces involved in the compression phase are not equal to the forces during the expansion phase. The loss in energy can be taken into account through the coefficient of restitution, $e$, which relates the relative velocity along the line of impact after the collision to that before,

$$
e=\left(v_{b f n}-v_{c f n}\right) / v_{c i} \cos \theta .
$$

Cochran and Stobbs gave a value of 0.67 for $e$, for a well hit drive, although no specifics of how this value was obtained were given. Measurements of golf balls fired into a station- ary plate have given higher values for $e$. For example, Lieberman and Johnson ${ }^{11}$ give values for $e$ decreasing from approximately 0.76 for impact speeds of $37 \mathrm{~m} / \mathrm{s}$ to values of around 0.72 for impact speeds of $50 \mathrm{~m} / \mathrm{s}$. Applying a linear fit to their results gives the following empirical equation for the dependence of $e$ on the initial clubhead speed and loft,

$$
e=0.86-0.0029 v_{c i} \cos \theta,
$$

and this equation will be used in the subsequent calculations.

After contact with the clubhead, the golf ball will not only begin to compress but will also start to slide up along the face of the clubhead. The friction between the ball and the clubface will turn this sliding motion into a rolling motion. Both Cochran and Stobbs and Chou et al. ${ }^{12}$ found that the initial spin of the launched ball was independent of the surface of the clubface for the typical loft angles that are found with drivers. This indicates that the ball is in a pure rolling state when it leaves the face of the clubhead, in which case

$$
v_{b f p}-v_{c f p}=\omega_{b f} r .
$$

Solving Eqs. (4) and (6) for $v_{c f n}$ and $v_{c f p}$, respectively, and substituting these expressions into Eqs. (1) and (2) results in the following expressions for the launch velocity components of the golf ball;

$$
v_{b f n}=(1+e) v_{c i} \cos \theta /(1+m / M),
$$

and

$$
v_{b f p}=-v_{c i} \sin \theta /\left(1+m / M+m r^{2} / I\right) .
$$

The launch speed and launch angle of the golf ball are then given by

$$
v_{b o}=v_{b f}=\left(v_{b f n}^{2}+v_{b f p}^{2}\right)^{1 / 2},
$$

and

$$
\psi_{b o}=\theta+\tan ^{-1}\left(v_{b f p} / v_{b f n}\right) .
$$

The spin of the golf ball when it leaves the face of the driver is determined by Eq. (3),

$$
\omega_{b o}=\omega_{b f}=-m v_{b f p} r / I .
$$

A standard USGA approved golf ball has a diameter of $4.27 \mathrm{~cm}\left(1.68^{\prime \prime}\right)$ and a mass of $45.9 \mathrm{~g}(1.62 \mathrm{oz})$. Using these values along with a mass of $200 \mathrm{~g}$ and an impact speed of 45 $\mathrm{m} / \mathrm{s}$ for the clubhead, which are typical values for a drive, Eqs. (7)-(11) were used to determine how changing the loft of the clubhead changes the launch speed, launch angle, and the initial spin of the golf ball. The results are shown in Fig. 2. As is seen, the launch speed of the golf ball decreases with increasing loft while the initial spin of the ball increases.

\section{B. Dynamic loft of the clubhead}

The loft, $\theta$, used in the theoretical model of the impact and in Fig. 2, is the angle the clubface makes with the vertical when the clubhead impacts with the golf ball. This angle is properly referred to as the dynamic loft of the clubhead. The dynamic loft, in general, is not equal to the clubface loft which is typically specified on the golf club. The clubface loft is the angle the clubface makes with the vertical when the sole of the clubhead is at rest on the ground. The difference between the clubface loft and the dynamic loft is due to the flexing of the golf club shaft.

The motion of a golf club shaft during a swing is similar to the motion of a whip. Initially, at the top of the swing, due 


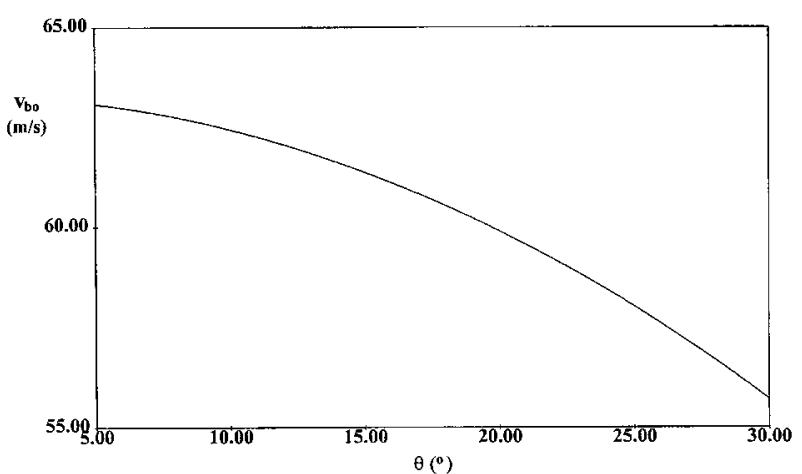

a)

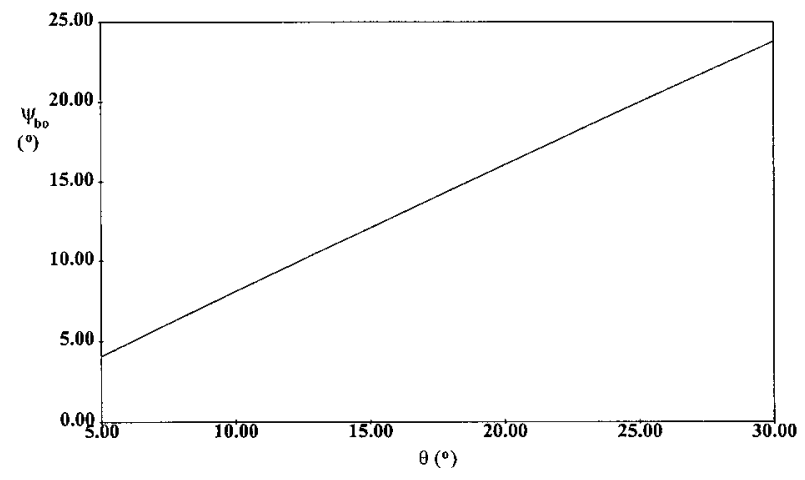

b)

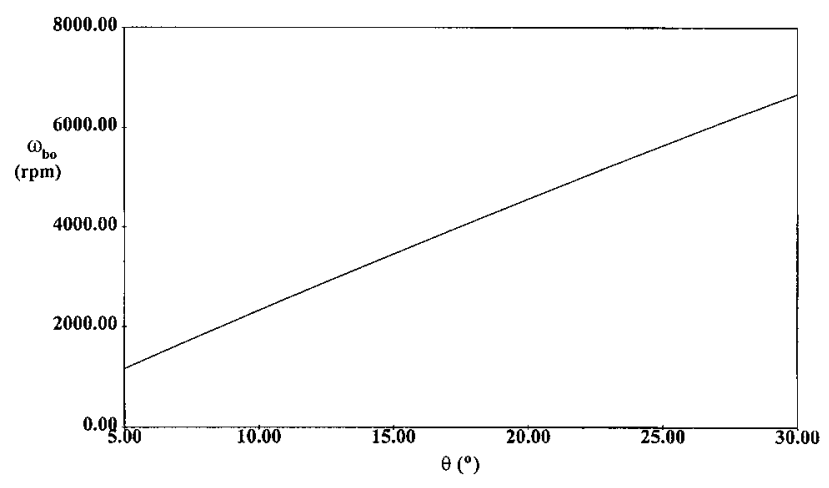

c)

Fig. 2. The dependence of the golf ball's (a) launch speed $\left(v_{b o}\right)$, (b) launch angle $\left(\psi_{b o}\right)$, and $(\mathrm{c})$ initial spin $\left(\omega_{b o}\right)$ on the loft $(\theta)$ of the clubhead.

to the inertia of the clubhead, the shaft will bend back so as to leave the clubhead behind. However, the clubhead not only catches up, but the shaft flexes forward such that at impact the clubhead is several degrees ahead of the position it would have had if the shaft had not flexed. Jorgensen photographed a particular swing of a professional golfer and measured the actual angular deflection of the clubhead prior to impact to be $3.3^{\circ}$. The dynamic loft is therefore approximately $3.3^{\circ}$ greater than the clubface loft in this case. The flex of a given golf club shaft will depend on the material, size, and shape of the shaft and on the mass and velocity of the clubhead. However, in general, the dynamic loft will be several degrees greater than the clubface loft.

\section{Trajectory and carry of the golf ball}

Once the golf ball leaves the face of the clubhead its motion will be determined by the forces due to gravity and the

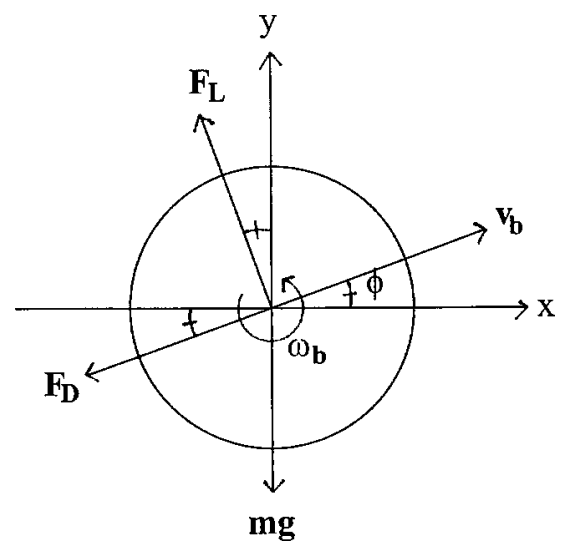

Fig. 3. The forces acting on a golf ball as it travels through the air with velocity $v_{b}$ and backspin $\omega_{b}$.

air. The force due to the air can be resolved into a component which acts parallel to the direction of motion, the drag, and a component perpendicular to the velocity vector, the lift. Figure 3 indicates these forces acting on a golf ball traveling at a speed $v_{b}$ with backspin $\omega_{b}$ in a direction of $\phi$ with respect to the horizontal.

The drag, $F_{D}$, on a ball of radius $r$ traveling through a fluid of density $\rho, 1.204 \mathrm{~kg} / \mathrm{m}^{3}$ for dry air at $20^{\circ} \mathrm{C}$, and at a speed $v_{b}$ is given by

$$
F_{D}=\frac{1}{2} \rho\left(\pi r^{2}\right) C_{D} v_{b}^{2}
$$

where $C_{D}$ is the drag coefficient. The drag coefficient depends on the Reynolds number, the nature of the ball's surface, and in general on the speed of the ball and its spin. Similarly the lift, $F_{L}$, acting on the golf ball is given by

$$
F_{L}=\frac{1}{2} \rho\left(\pi r^{2}\right) C_{L} v_{b}^{2},
$$

where $C_{L}$ is the lift coefficient. The lift and drag coefficients measured by Bearman and Harvey were found in general to decrease with increasing speed and to increase with increasing spin. Using these coefficients, Bearman and Harvey calculated the carry of golf balls for the same launch parameters that were obtained from high speed photographs of balls hit by a driving machine. Good agreement was found between the calculated golf ball carries and the measured carries for hexagonally dimpled balls and therefore it is these drag and lift coefficients that will be used in this paper. Given these coefficients, the motion of the ball follows from Newton's second law applied to the $x$ and $y$ axes of Fig. 3:

$$
a_{x}=\left(-F_{D} \cos \phi-F_{L} \sin \phi\right) / m,
$$

and

$$
a_{y}=\left(-F_{D} \sin \phi+F_{L} \cos \phi\right) / m-g,
$$

where $g$, the acceleration due to gravity, will be taken to be $9.81 \mathrm{~m} / \mathrm{s}^{2}$.

The rate at which the spin of the golf ball decays, or the angular acceleration, has been determined in wind tunnel tests. These tests indicate that the decay is approximately exponential with the spin rate on landing for a driver shot estimated to be approximately $75 \%$ of the initial spin rate. The following empirical equation for the angular acceleration, $\alpha$, of a golf ball, obtained from the results of Smits and Smith, ${ }^{13}$ will be used in the calculations, 


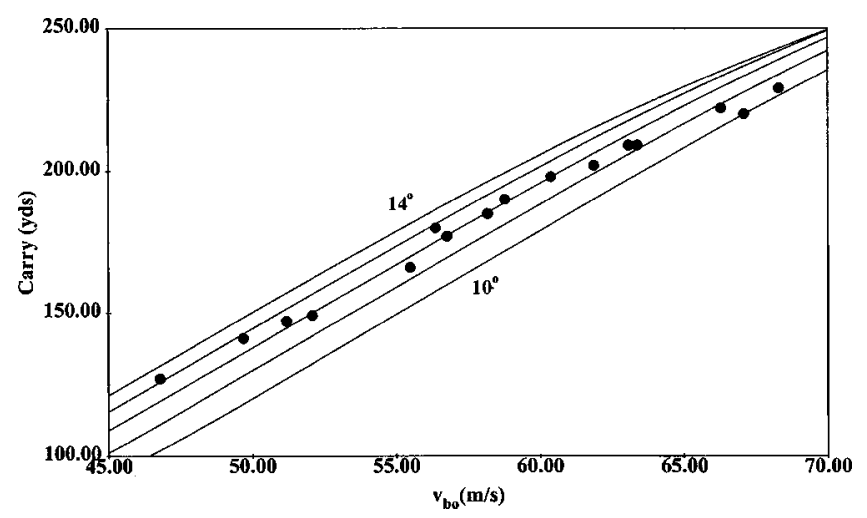

Fig. 4. Theoretical values for the carry (_- of a golf ball for dynamic lofts ranging from 10 to 14 in steps of 1 versus the launch velocity $\left(v_{b o}\right)$ of the golf ball compared with the experimental carry results of Williams $(\bullet)$.

$$
\alpha=-(0.00002)\left(\omega_{b} v_{b} / r\right) \text {. }
$$

Williams ${ }^{14}$ provided data on the carry and drive of a British golf ball hit with a driving machine. British golf balls have the same mass, $45.9 \mathrm{~g}$, as a standard golf ball, but have a slightly smaller diameter of $4.12 \mathrm{~cm}$. The results Williams presented gave the carry and total drive for various measured launch speeds of the golf ball. In order to compare the above theoretical model of the impact and trajectory of a golf ball with Williams' data, the carry distances for a clubhead mass of $200 \mathrm{~g}$ and for various clubhead speeds and dynamic lofts were determined. The golf ball's launch speed, launch angle, and initial spin were calculated using Eqs. (7)-(11). These launch parameters were then used along with interpolated $C_{D}$ and $C_{L}$ values from Bearman and Harvey to compute the trajectories of the golf balls using Eqs. (12)-(16). The resulting carry versus the launch speed of the golf ball is given for various dynamic lofts in Fig. 4 along with the carry results of Williams. The trajectories were calculated numerically using a step size of $0.001 \mathrm{~s}$, which resulted in a calculation uncertainty in the carry and drive of less than $0.1 \mathrm{yd}$. The distances on this figure and following ones will be given in yards, as this is the standard unit used in the game. As is seen, reasonable agreement with Williams' data is obtained for a dynamic loft of approximately $12^{\circ}$. Williams did not specify the value of the clubface loft of the driver used in the tests; however, it is expected that a standard commercial driver with a clubface loft of approximately $10^{\circ}$ was used. A dynamic loft of approximately $12^{\circ}$ would therefore seem to be a reasonable result.

\section{Run of the golf ball}

After impact with the ground, the golf ball will ideally bounce several times and then roll to a stop, the total distance traveled being referred to as the run. In practice the motion of the golf ball after landing can be fairly erratic as it depends on the condition and slope of the fairway, especially at the landing point. For the purposes of this paper the general dependence of the run on the landing velocity and spin of the golf ball needs to be determined. This will require both phases of the run, the bouncing and then the subsequent roll, to be modeled, although it must be stated that any model can only roughly approximate the general run of a golf ball.

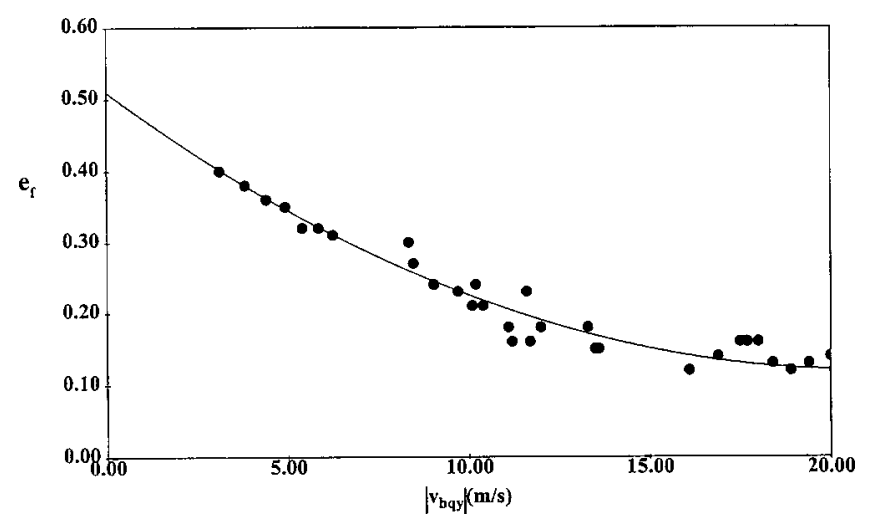

Fig. 5. Experimental values $(\bullet)$ of the coefficient of restitution $\left(e_{f}\right)$ versus the magnitude of the landing velocity $\left(\left|v_{b q y}\right|\right)$ along with Eq. (19).

Daish considered the case of a ball bouncing on a plane solid surface and his model will be used to determine the overall bounce length. The length of the first bounce, $b_{1}$, will be given by

$$
b_{1}=(2 / g) v_{b r x} v_{b r y},
$$

where $v_{b r x}$ and $v_{b r y}$ are the velocity components of the golf ball after rebound. The vertical component of the rebound velocity can be expressed as

$$
v_{b r y}=e_{f}\left|v_{b q y}\right|,
$$

where $v_{b q y}$ is the vertical component of the landing velocity and $e_{f}$ is the coefficient of restitution between the golf ball and the fairway. The value of $e_{f}$ will in general depend on the nature of the fairway and on the landing velocity of the golf ball. In order to determine the dependence of $e_{f}$ on the landing velocity, measurements were made of the bounce height of golf balls which were either dropped from specific heights onto a grass lawn or tossed vertically upward with their times of flight recorded. The resulting coefficients of restitution plotted against the calculated magnitudes of the landing velocities are shown in Fig. 5. As is seen, the coefficient of restitution decreases with increasing impact velocity. The following equation, which is also plotted in Fig. 5,

$$
e_{f}=0.510-0.0375\left|v_{b q y}\right|+0.000903\left|v_{b q y}\right|^{2},
$$

fits well with the experimental results and will be used in the subsequent calculations of the bounce length.

Daish found that, for the case applicable to a drive, the coefficient of friction will, in general, be great enough to arrest the backspin of the golf ball on impact with the ground and to put the ball in a state of rolling when it rebounds from the surface. In this case the horizontal component of the rebound velocity was shown to be given by

$$
v_{b r x}=\left(5 v_{b q x}-2 r \omega_{b q}\right) / 7,
$$

where $v_{b q x}$ is the horizontal component of the landing velocity and $\omega_{b q}$ is the backspin of the golf ball at impact. Daish also showed that for impact on a plane solid surface, the horizontal velocity component of the golf ball will stay constant through each subsequent bounce. Therefore each bounce will be $e_{f}$ times the length of the previous one so the total bounce length, $b_{T}$, of the golf ball will be

$$
b_{T}=\left(1+e_{f 2}+e_{f 2} e_{f 3}+e_{f 2} e_{f 3} e_{f 4}+\cdots\right) b_{1},
$$




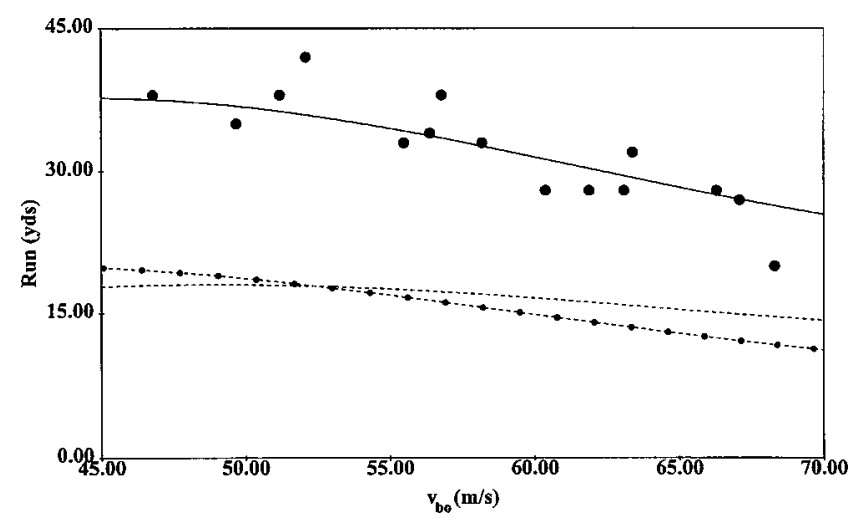

Fig. 6. Theoretical values for the bounce (---), roll (-•-), and run (- $\square$ ) of a golf ball, for $\mu_{r}=1.0$, versus the launch speed $\left(v_{b o}\right)$ of the golf ball compared with the experimental run results of Williams $(\bullet)$.

where $e_{f i}$ is the coefficient of restitution for the $i$ th bounce. After the first bounce, the vertical component of the rebound velocities will be relatively small, less than $3 \mathrm{~m} / \mathrm{s}$, for the initial golf ball launch speeds considered. Therefore, $e_{f}$ will be approximately equal to 0.5 for the second and subsequent bounces. Therefore,

$$
\begin{aligned}
b_{T} & =\left(1+(0.5)+(0.5)^{2}+(0.5)^{3}+\cdots\right) b_{1} \\
& =2 b_{1},
\end{aligned}
$$

and the total bounce length is approximately twice the initial bounce length.

After the bounce phase, the golf ball will be rolling along the ground with a speed of $v_{b r x}$ and, assuming a constant frictional force of $\mu_{r} m g$, where $\mu_{r}$ can be identified as the coefficient of rolling friction, the roll distance will be given by

$$
r_{T}=v_{b r x}^{2} /\left(2 \mu_{r} g\right) .
$$

The total run, $\Lambda$, will therefore be given by

$$
\Lambda=b_{T}+r_{T} \text {. }
$$

To compare this model of the run with Williams' data, the landing velocity and spin were calculated for a golf ball struck by a $200 \mathrm{~g}$ clubhead, with a dynamic loft of $12^{\circ}$, and various clubhead speeds. The run was then calculated using Eqs. (17)-(24) and the theoretical dependence of the run as a function of the initial launch speed of the golf ball was determined. The coefficient of rolling friction, $\mu_{r}$, was used to fit the run distance obtained from the model to the results of Williams. Good agreement is found with Williams' data for a value of $\mu_{r}$ of approximately 1.0. Figure 6 shows the results with Williams' data along with the modeled bounce length, roll, and total run, for a value of $\mu_{r}$ of 1.0, plotted as a function of the launch speed of the golf ball. As is shown, the run decreases with increasing launch speed of the golf ball. The reason is that although the landing speed increases with increasing launch speed, the horizontal component of the landing velocity, $v_{b q x}$, decreases with increasing launch speed as the golf ball comes in at steeper angles.

Although the above model matches well with Williams' run data for the given value of $\mu_{r}$, there are differences with observations made of the behavior of bouncing golf balls on fairways. The most obvious difference is the height of the bounces. Using calculated values of $v_{b q y}$ for typical drives,

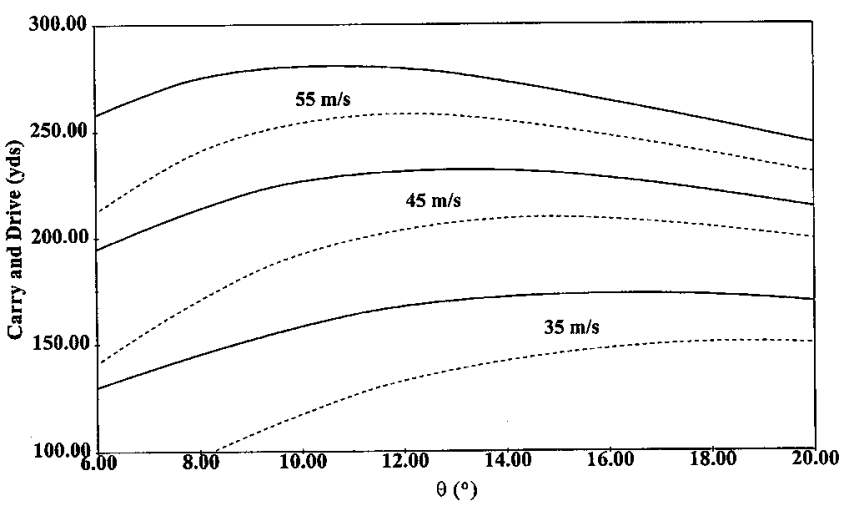

Fig. 7. The carry (---) and drive (_- ) of a golf ball versus the dynamic loft $(\theta)$ of a $200 \mathrm{~g}$ clubhead for various initial clubhead speeds $\left(v_{c i}\right)$.

the above model predicts initial bounce heights of only approximately $30 \mathrm{~cm}$ while observations indicate initial bounce heights of over $1 \mathrm{~m}$. The reason for the difference, as Daish pointed out in the case of the bounce of a cricket ball, is that the landing surface is not solid and the ground is deformed at impact. The ball is therefore actually rebounding from an inclined surface. The result is that the ball will be thrown higher in the air with an increased vertical velocity component, $v_{b r y}$, and will have its horizontal velocity component, $v_{b r x}$, reduced at each bounce. However, for the purposes of this paper, the above run model, which is fitted to Williams' data through the coefficient $\mu_{r}$, provides a reasonable dependence of the run on the landing velocity components.

\section{RESULTS}

Using the above models for the impact with the clubhead, the trajectory, and the run of a golf ball, the dependence of the carry and drive distances on the dynamic loft of a driver can be determined. The results for a standard golf ball and a $200 \mathrm{~g}$ clubhead for initial clubhead speeds of $35 \mathrm{~m} / \mathrm{s}(78$ $\mathrm{mph}), 45 \mathrm{~m} / \mathrm{s}(100 \mathrm{mph})$, and $55 \mathrm{~m} / \mathrm{s}(123 \mathrm{mph})$, corresponding to short, average, and long drives, are shown in Fig. 7. As is shown, the peaks of the curves are broad, with variances in the loft of $3^{\circ}$ resulting in a loss in the carry or the drive of only approximately $5 \mathrm{yd}$. Also, as is seen, the loft resulting in the maximum drive distance is a few degrees less than the loft which results in the maximum carry. For ex-

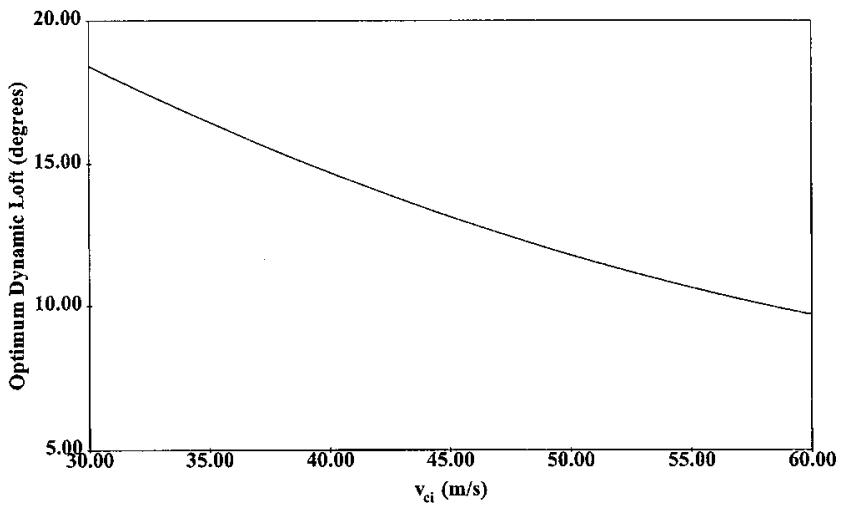

Fig. 8. The optimum dynamic loft of a $200 \mathrm{~g}$ clubhead versus initial clubhead speed $\left(v_{c i}\right)$. 


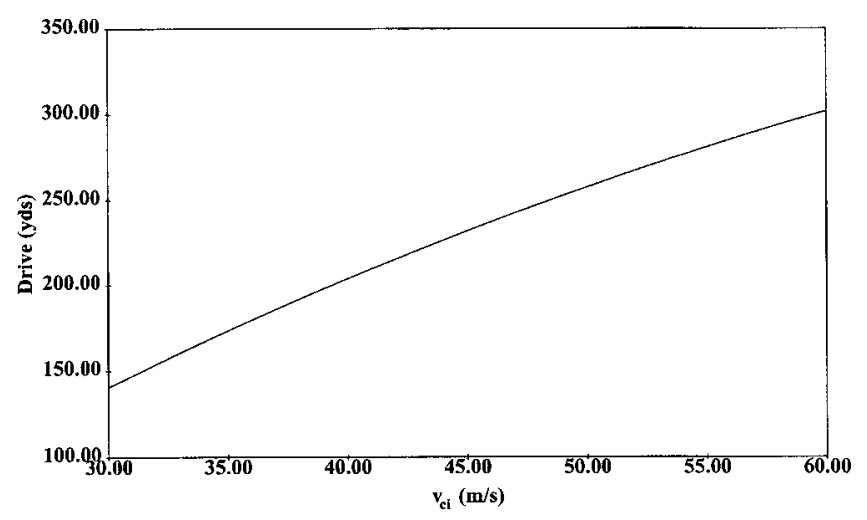

Fig. 9. The drive distance for the optimum dynamic loft versus initial clubhead speed $\left(v_{c i}\right)$.

ample, for an initial clubhead speed of $45 \mathrm{~m} / \mathrm{s}$, the dynamic loft which leads to maximum carry, $209 \mathrm{yds}$, is $14.9^{\circ}$ while the dynamic loft which leads to the maximum drive, $232 \mathrm{yds}$, is $13.1^{\circ}$. This difference is a result of the horizontal component of the landing velocity, $v_{b q x}$, being greater at smaller lofts and therefore leading to greater runs and subsequently greater drive distances.

The dynamic loft of the clubhead which results in the maximum drive distance is shown as a function of the clubhead speed at impact in Fig. 8. As is seen, the greater the clubhead speed, the lower the optimum dynamic loft. For example, for clubhead speeds of 35,45 , and $55 \mathrm{~m} / \mathrm{s}$, the optimum dynamic lofts are $16.5^{\circ}, 13.1^{\circ}$, and $10.7^{\circ}$, respectively. The dependence of the optimum loft on clubhead speed agrees, in general, with current practices in golf. For example, the manufacturer of one popular driver ${ }^{15}$ recommends a clubface loft of $11.5^{\circ}$ for clubhead speeds up to 36 $\mathrm{m} / \mathrm{s}$ decreasing to clubface lofts as low as $7^{\circ}$ for clubhead speeds in excess of $45 \mathrm{~m} / \mathrm{s}$.

The drive distance, corresponding to the optimum dynamic loft, is shown plotted against the clubhead speed in Fig. 9. As is shown, the maximum drive distance increases from $140 \mathrm{yds}$ for a clubhead speed of $30 \mathrm{~m} / \mathrm{s}$ to approximately $300 \mathrm{yds}$ for a clubhead speed of $60 \mathrm{~m} / \mathrm{s}$.

\section{CONCLUSION}

The models of the impact of a golf ball with a clubhead and the subsequent carry of the ball agree with available experimental results. The parametric fit of the run model with Williams' data gives a reasonable result for the general behavior of a golf ball after landing. These models allow the question of what clubhead loft will lead to the maximum drive distance to be answered. The optimum dynamic lofts for drivers that were determined agree reasonably well with the lofts of drivers used in the game.

Further analysis of the problem would require additional data on the relationship between the clubface loft and the dynamic loft. Also, experimental measurements on the individual contributions of the bounce and the roll to the run of a golf ball and their dependence on the landing velocity components would be required.

The results presented in this paper will hopefully be of interest to those students and colleagues who not only enjoy an interesting and practical physics problem but who also may find themselves at their local golf course.

${ }^{1}$ R. K. Adair, The Physics of Baseball (Harper-Collins, New York, 1994), 1 st ed.

${ }^{2} \mathrm{C}$. Frohlich, "Aerodynamic drag crisis and its possible effect on the flight of baseballs," Am. J. Phys. 52 (4), 325-334 (1984).

${ }^{3}$ P. J. Brancazio, "'Trajectory of a fly ball,"' Phys. Teach. 23, 20-23 (1985).

${ }^{4}$ A. F. Rex, "The effect of spin on the flight of batted baseballs," Am. J. Phys. 53 (11), 1073-1075 (1985).

${ }^{5} \mathrm{H}$. Erlichson, "Maximum projectile range with drag and lift, with particular application to golf," Am. J. Phys. 51 (4), 357-361 (1983).

${ }^{6}$ P. W. Bearman and J. K. Harvey, "Golf Ball Aerodynamics,' Aeronaut. Q. 27, 112-122 (1976).

${ }^{7}$ W. M. MacDonald and S. Hanzely, "The physics of the drive in golf," Am. J. Phys. 59 (3), 213-218 (1991).

${ }^{8}$ A. Cochran and J. Stobbs, The Search for the Perfect Swing (Lippincott, New York, 1968).

${ }^{9}$ C. B. Daish, The Physics of Ball Games (English Universities, London, 1972).

${ }^{10}$ T. P. Jorgensen, The Physics of Golf (Springer-Verlag, New York, 1994).

${ }^{11}$ B. B. Lieberman and S. H. Johnson, "An analytical model for ball-barrier impact,"' Proceedings of the 1994 World Scientific Congress of Golf, edited by A. J. Cochran and M. R. Farrally (E \& FN Spon, London, 1994).

${ }^{12}$ P. C. Chou, D. Liang, J. Yang, and W. Gobosh, "Contact forces, coefficient of restitution, and spin rate of golf ball impact," Proceedings of the 1994 World Scientific Congress of Golf, edited by A. J. Cochran and M. R. Farrally (E \& FN Spon, London, 1994).

${ }^{13}$ A. J. Smits and D. R. Smith, "A new aerodynamic model of a golf ball in flight," in Proceedings of the 1994 World Scientific Congress of Golf, edited by A. J. Cochran and M. R. Farrally (E \& FN Spon, London, 1994).

${ }^{14} \mathrm{D}$. Williams, "Drag force on a golf ball in flight and its practical significance,’ Q. J. Mech. Appl. Math. XII (3), 387-392 (1959).

${ }^{15}$ The data were obtained from the Ping company web site: www.pinggolf.com 